\title{
New Multiple Solution to the Boussinesq Equation and the Burgers-Like Equation
}

\author{
Hasan Bulut, Münevver Tuz, and Tolga Akturk \\ Department of Mathematic, Faculty of Science and Arts, University of Firat, 23119 Elaziğ, Turkey \\ Correspondence should be addressed to Hasan Bulut; hbulut@firat.edu.tr \\ Received 3 February 2013; Accepted 3 April 2013 \\ Academic Editor: Mamdouh M. El Kady
}

Copyright (C) 2013 Hasan Bulut et al. This is an open access article distributed under the Creative Commons Attribution License, which permits unrestricted use, distribution, and reproduction in any medium, provided the original work is properly cited.

By considering an improved tanh function method, we found some exact solutions of Boussinesq and Burgers-like equations. The main idea of this method is to take full advantage of the Riccati equation which has more new solutions. We found some exact solutions of the Boussinesq equation and the Burgers-like equation.

\section{Introduction}

In recent years, nonlinear phenomena play a crucial role in applied mathematics and physics. Directly searching for exact solutions of nonlinear partial differential equations (PDEs) has become more and more attractive partly due to the availability of computer symbolic systems like Mathematica or Maple that allow us to perform some complicated and tedious algebraic calculation on a computer as well as help us to find exact solutions of PDEs [1-5] now.

Many explicit exact methods have been introduced in the literature [6-14]. Some of them are Painlevé method, homogeneous balance method, similarity reduction method, sinecosine method, Darboux transformation, Cole-Hopf transformation, generalized Miura transformation, tanh method, Backlund transformation, and others methods $[15,16]$.

One of the most effectively straightforward methods constructing exact solution of PDEs is the extended tanh function method [17]. Let us simply describe the tanh function. For doing this, one can consider in two variables general form of nonlinear PDE as follows:

$$
H\left(u, u_{t t}, u_{x}, u_{x x}, \ldots\right)=0
$$

and transform (1) with

$$
u(x, t)=u(\xi), \quad \xi=k(x-\lambda t),
$$

where $k$ and $\lambda$ are the wave number and wave speed, respectively. After the transformation, we get a nonlinear ODE for $u(\xi)$ as follows:

$$
H^{\prime}\left(u^{\prime}, u^{\prime \prime}, u^{\prime \prime \prime}, \ldots\right)=0 .
$$

The fact that the solutions of many nonlinear equations can be expressed as a finite series of tanh functions that motivates us to seek for the solutions of (3) in the form

$$
u(x, t)=u(\xi)=\sum_{i=0}^{m} a_{i} \tanh ^{i}(\xi)=\sum_{i=0}^{m} a_{i} F^{i},
$$

where $F^{i}=\tanh ^{i}(\xi)$, an equation for $F(\xi)$, is obtained. $m$ is a positive integer that can be determined by balancing the linear term of highest order with the nonlinear term in (1), and $k, \lambda, a_{1}, a_{2}, \ldots, a_{m}$ are parameters to be determined.

Substituting solution (4) into (3) yields a set of algebraic equations for $F^{i}$, then all coefficients of $F^{i}$ have to vanish. From these relations $k, \lambda, a_{1}, a_{2}, \ldots, a_{m}$ can be determined.

In this work, we will consider to solve general Boussinesq equation and the Burgers-like equation by using the improved tanh function method which is introduced by Chen and Zhang [18].

\section{Method and Its Applications}

The main idea of this method is to take full advantage of the Riccati equation that tanh function satisfies and uses its 
solutions $F$ to replace $\tanh \xi$. The required Riccati equation is given as

$$
F^{\prime}=A+B F+C F^{2},
$$

where $F=(d F / d \xi)$ and $A, B$, and $C$ are constant. In the following, Chen and Zhang [18] have given several cases to get the solution of (5) in the form of finite series of tanh functions (4).

Case 1. If $C=0, B \neq 0$, then (5) has the solutions

$$
\frac{\exp (B \xi)-A}{B} \text {. }
$$

Case 2. If $A=1 / 2, B=0$, and $C=-1 / 2$, then (5) has the solutions $\cot h \xi \pm \csc h \xi, \tan h \xi \pm i \sec h \xi\left(i^{2}=-1\right)$.

Case 3. If $A=C= \pm 1 / 2, B=0$, then (5) has the solutions $\sec \xi \pm \tan \xi, \csc \xi \pm \cot \xi$.

Case 4. If $A=1, B=0$, and $C=-1$, then (5) has the solutions $\tan h \xi$, $\cot h \xi$.

Case 5. If $A=C=1, B=0$, then (5) has the solutions $\tan \xi$.

Case 6. If $A=C=-1, B=0$, then (5) has the solutions $\cot \xi$.

Case 7. If $A=B=0, C \neq 0$, then (5) has the solutions $-1 /(c \xi+$ $\left.c_{0}\right)$.

The solutions of (1) can be expressed in the form

$$
u(x, t)=u(\xi)=\sum_{i=0}^{m} a_{i} F^{i},
$$

where $\xi=k x-k \omega t, k$ and $\omega$ are the wave number and the wave speed, respectively, $n$ is a positive integer that can be determined by balancing the linear term of highest order with the nonlinear term in (1), and $a_{0}, a_{1}, \ldots, a_{n}$ are parameters to be determined.

Introducing the similarity variable $\xi=k x-k \omega t$, the travelling wave solutions $u(\xi)$ satisfy

$$
H^{\prime}\left(u^{\prime}, u^{\prime \prime}, u^{\prime \prime \prime}, \ldots\right)=0 .
$$

Balancing the highest order of linear term with nonlinear term in (3), we can determine $n$ in (4).

We illustrate the method by considering the Boussinesq equation and Burgers-like equtions.

Example 1 (The Boussinesq equation). Let's consider

$$
u_{t t}=\left(c_{1} u+c_{2} u^{n}\right)_{x x}+u_{x x t t}=0 .
$$

If we accept that $c_{1} \geq 0, c_{2} \leq 0$ and $n=2, c=-1$, we conclude (10) by (9) as follows:

$$
u_{t t}-u_{x x}+2 u \cdot u_{x x}-u_{x x t t}=0
$$

for doing this example. We could use transformation with (1) for the Boussinesq equation. Let us consider the Boussinesq equation as follows:

$$
u(x, t)=u(\xi), \quad \xi=k x-k w t .
$$

Substituting (11) into (10), we get

$$
w^{2} u^{\prime \prime}-u^{\prime \prime}+2\left(u^{\prime}\right)^{2}+2 u \cdot u^{\prime \prime}-k^{2} w^{2} u^{4}=0,
$$

and integrating (12) we deduce the following equation:

$$
w^{2} u^{\prime}-u^{\prime}+2 u \cdot u^{\prime}-k^{2} w^{2} u^{\prime \prime \prime}=0 .
$$

Balancing $\left(u \cdot u^{\prime}\right)$ with $\left(u^{\prime \prime \prime}\right)$ gives $m=2$. Therefore, we may choose

$$
u=a_{0}+a_{1} F+a_{2} F^{2} .
$$

Substituting (14) into (13) along with (5) and using Mathematica yield a system of equations $w, t$, and $F^{M}$. Setting the coefficients of $F^{M}$ in the obtained system of equations to zero, we can deduce the following set of algebraic polynomials with respect to unknowns $a_{0}, a_{1}$, and $a_{2}$

$$
\begin{aligned}
& u^{\prime}= a_{1} A+a_{1} B F+a_{1} C F^{2} \\
&+2 a_{2} A F+2 a_{2} B F^{2}+2 a_{2} C F^{3}=0, \\
& u \cdot u^{\prime}= a_{0} a_{1} A+a_{0} a_{1} B F+a_{0} a_{1} C F^{2} \\
&+2 a_{0} a_{2} A F+2 a_{0} a_{2} B F^{2}+2 a_{0} a_{2} C F^{3} \\
&+a_{1}^{2} A F+a_{1}^{2} B F^{2}+a_{1}^{2} C F^{3} \\
&+2 a_{1} a_{2} A F^{2}+2 a_{1} a_{2} B F^{3} \\
&+2 a_{1} a_{2} C F^{4}+a_{1} a_{2} A F^{2} \\
&+a_{1} a_{2} B F^{3}+a_{1} a_{2} C F^{4} \\
&+2 a_{2}^{2} A F^{3}+2 a_{2}^{2} B F^{4}+2 a_{2}^{2} C F^{5}, \\
& u^{\prime \prime \prime}= a_{1} B^{2} A+a_{1} B^{3} F+7 a_{1} B^{2} C F^{2} \\
&+ 8 a_{1} A B C F+12 a_{1} B C^{2} F^{3}+2 a_{1} A^{2} C \\
&+ 8 a_{1} A C^{2} F^{2}+6 a_{1} C^{3} F^{4}+6 a_{2} A^{2} B \\
&+ 14 a_{2} A B^{2} F+52 a^{2} A B C F^{2} \\
&+ 16 a_{2} A^{2} C F+40 a_{2} A C^{2} F^{3} \\
&+ 8 a_{2} B^{3} F^{2}+38 a^{2} B^{2} C F^{3} \\
&+ 54 a_{2} B C^{2} F^{4}+24 a_{2} C^{3} F^{5}, \\
& F^{0}: w^{2} a_{1} A-a_{1} A+2 a_{0} a_{1} A-k^{2} w^{2} a_{1} B^{2} A \\
&- 2 k_{1}^{2} w^{2} a_{1} A^{2} C-6 k^{2} w^{2} a_{2} A^{2} B=0, \\
&+ 2 a_{0} a_{1} B+4 a_{0} A-a_{1} B-2 a_{2} A+2 a_{1}^{2} A-k^{2} w^{2} a_{1} B^{3} \\
&- 8 k^{2} w^{2} a_{1} A B C-14 k^{2} w^{2} a_{2} A B^{2} \\
&- 16 k^{2} w^{2} a_{2} A^{2} C=0, \\
& \\
&
\end{aligned}
$$




$$
\begin{gathered}
F^{2}: w^{2} a_{1} C+2 w^{2} a_{2} B-a_{1} C-2 a_{2} B \\
+2 a_{0} a_{1} C+4 a_{0} a_{2} B+2 a_{1}^{2} B \\
+6 a_{1} a_{2} A-7 k^{2} w^{2} a_{1} B^{2} C-8 k^{2} w^{2} a_{1} A C^{2} \\
-52 k^{2} w^{2} A B C-8 k^{2} w^{2} a_{2} B^{3}=0, \\
F^{3}: 2 w^{2} a_{2} C-2 a_{2} C+4 a_{0} a_{2} C+2 a_{1}^{2} C \\
+4 a_{1} a_{2} B+2 a_{1} a_{2} B+4 a_{2}^{2} A-12 k^{2} w^{2} a_{1} B C^{2} \\
-40 k^{2} w^{2} a_{2} A C^{2}-38 k^{2} w^{2} B^{2} C=0, \\
F^{4}:-6 a_{1} a_{2} C+4 a_{2}^{2} B-6 k^{2} w^{2} a_{1} C^{3}-54 k^{2} w^{2} a_{2} B C^{2}=0, \\
F^{5}: 4 a_{2}^{2} C-24 k^{2} w^{2} a_{2} C^{3}=0 .
\end{gathered}
$$

From the solutions of the system, we can find

$$
\begin{gathered}
B=0, \quad a_{0}=4 k^{2} w^{2} A C-\frac{1}{2} w^{2}+\frac{1}{2}, \\
a_{1}=0, \quad a_{2}=6 k^{2} w^{2} C^{2}, \\
w=\frac{1}{4 k^{2} A C+1},
\end{gathered}
$$

and with the aid of Mathematica, we find the following.

(i) When we choose $A=1, B=0$, and $C=1$ in (16), then we can deduce

$$
a_{0}=4 k^{2} w^{2}-\frac{1}{2} w^{2}+\frac{1}{2}, \quad a_{2}=6 k^{2} w^{2} .
$$

Therefore, the solution can be found as

$$
u(x, t)=\left(4 k^{2} w^{2}-\frac{1}{2} w^{2}+\frac{1}{2}\right)+6 k^{2} w^{2}\left[\tan ^{2}(k x-k w t)\right] .
$$

(ii) In this case, if we take $A=-1, B=0$, and $C=-1$ in (16), then we have

$$
\begin{gathered}
a_{0}=4 k^{2} w^{2}-\frac{1}{2} w^{2}+\frac{1}{2}, \quad a_{2}=6 k^{2} w^{2}, \\
u(x, t)=\left(4 k^{2} w^{2}-\frac{1}{2} w^{2}+\frac{1}{2}\right)+6 k^{2} w^{2} \cot [k x-k w t] .
\end{gathered}
$$

(iii) Again, when we choose $A=1, B=0, C=-1$ then from (16) is

$$
a_{0}=-4 k^{2} w^{2}-\frac{1}{2} w^{2}+\frac{1}{2}, \quad a_{2}=6 k^{2} w^{2},
$$

$u(x, t)=\left(-4 k^{2} w^{2}-\frac{1}{2} w^{2}+\frac{1}{2}\right)+6 k^{2} w^{2} \tan h^{2}(k x-k w t)$,

$u(x, t)=\left(-4 k^{2} w^{2}-\frac{1}{2} w^{2}+\frac{1}{2}\right)+6 k^{2} w^{2} \cot h^{2}(k x-k w t)$. (iv) When we choose $A=(1 / 2), B=0$, and $C=(1 / 2)$, then we can find the coefficients of (16) as

$$
a_{0}=k^{2} w^{2}-\frac{1}{2} w^{2}+\frac{1}{2}, \quad a_{2}=\frac{3}{2} k^{2} w^{2},
$$

and using the coefficients, the solutions can be found as

$$
\begin{aligned}
u(x, t)= & \left(k^{2} w^{2}-\frac{1}{2} w^{2}+\frac{1}{2}\right)+\frac{3}{2} k^{2} w^{2} \\
& \times[\sec (k x-k w t)+\tan (k x-k w t)]^{2}, \\
u(x, t)= & \left(k^{2} w^{2}-\frac{1}{2} w^{2}+\frac{1}{2}\right)+\frac{3}{2} k^{2} w^{2} \\
& \times[\csc (k x-k w t)+\cot (k x-k w t)]^{2} .
\end{aligned}
$$

(v) When we choose $A=-(1 / 2), B=0$, and $C=-(1 / 2)$, then we can find the coefficients of (16) as follows:

$$
\begin{aligned}
a_{0}= & k^{2} w^{2}-\frac{1}{2} w^{2}+\frac{1}{2}, \quad a_{2}=\frac{3}{2} k^{2} w^{2}, \\
u(x, t)= & \left(k^{2} w^{2}-\frac{1}{2} w^{2}+\frac{1}{2}\right)+\frac{3}{2} k^{2} w^{2} \\
& \times[\sec (k x-k w t)+\tan (k x-k w t)]^{2}, \\
u(x, t)= & \left(k^{2} w^{2}-\frac{1}{2} w^{2}+\frac{1}{2}\right)+\frac{3}{2} k^{2} w^{2} \\
& \times[\csc (k x-k w t)+\cot (k x-k w t)]^{2} .
\end{aligned}
$$

(vi) When we choose $A=(1 / 2), B=0$, and $C=-(1 / 2)$, then we can find the coefficients of (16) as follows:

$$
\begin{aligned}
a_{0}= & -k^{2} w^{2}-\frac{1}{2} w^{2}+\frac{1}{2}, \quad a_{2}=\frac{3}{2} k^{2} w^{2}, \\
u(x, t)= & \left(-k^{2} w^{2}-\frac{1}{2} w^{2}+\frac{1}{2}\right)+\frac{3}{2} k^{2} w^{2} \\
& \times[\operatorname{coth}(k x-k w t) \pm \operatorname{csch}(k x-k w t)]^{2}, \\
u(x, t)= & \left(-k^{2} w^{2}-\frac{1}{2} w^{2}+\frac{1}{2}\right)+\frac{3}{2} k^{2} w^{2} \\
& \times[\tanh (k x-k w t) \pm i \operatorname{sech}(k x-k w t)]^{2} .
\end{aligned}
$$

(vii) When we choose $A=0, B=0$, and $C \neq 0$, then we can find the coefficients of (16) as follows:

$$
\begin{gathered}
a_{0}=-\frac{1}{2} w^{2}+\frac{1}{2}, \quad a_{2}=6 k^{2} w^{2} C^{2}, \\
u(x, t)=\left(-\frac{1}{2} w^{2}+\frac{1}{2}\right)+\frac{3}{2} k^{2} w^{2} C^{2}\left(-\frac{1}{(k x-k w t)+C_{0}}\right)^{2} .
\end{gathered}
$$

Figure 1 gives to us 2D and 3D graphics for (25).

Example 2 (Burger-like equations). Let's consider

$$
u_{t}+u_{x}+u u_{x}+\lambda u_{x x}=0,
$$




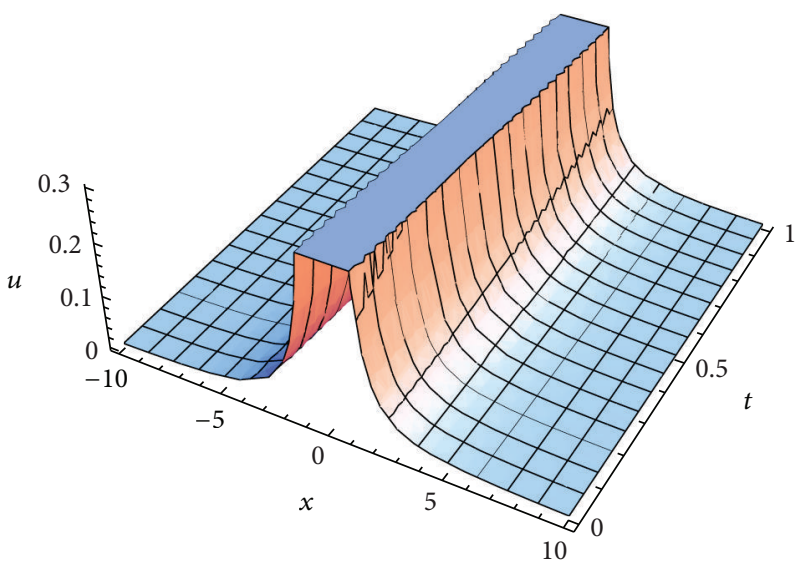

(a)

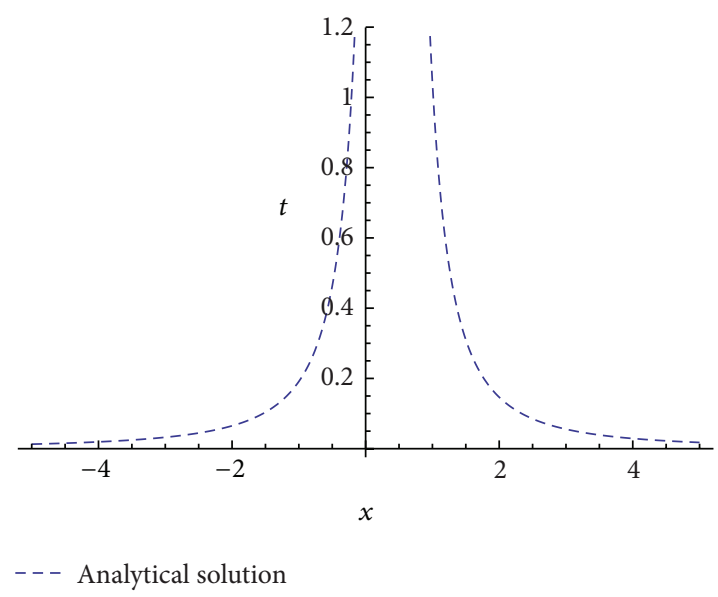

(b)

Figure 1: The solution (25) is shown at $k=w=1, C=0.5$, and $C_{0}=0.1$, and the second graph represents the exact analytical solution of (25) for $t=0.5$.

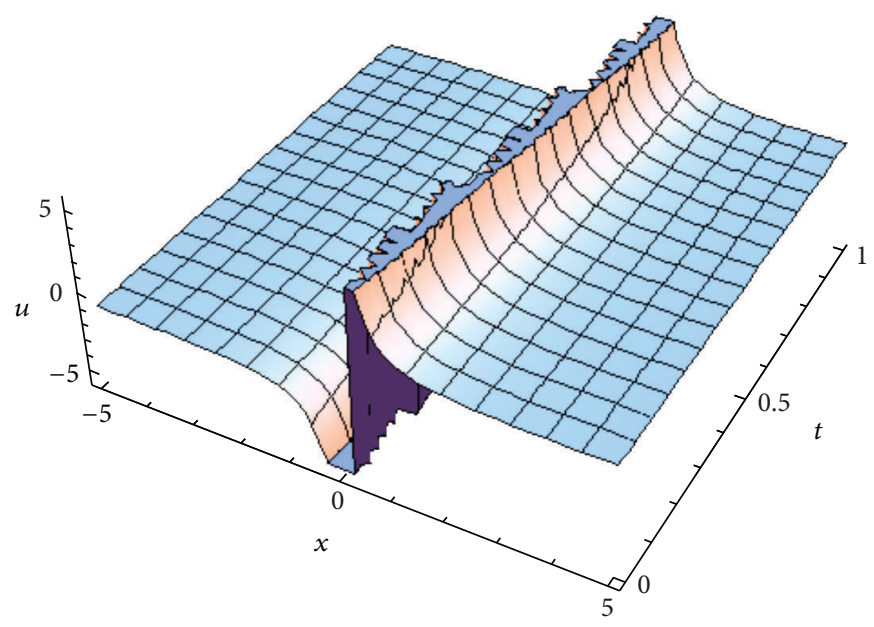

(a)

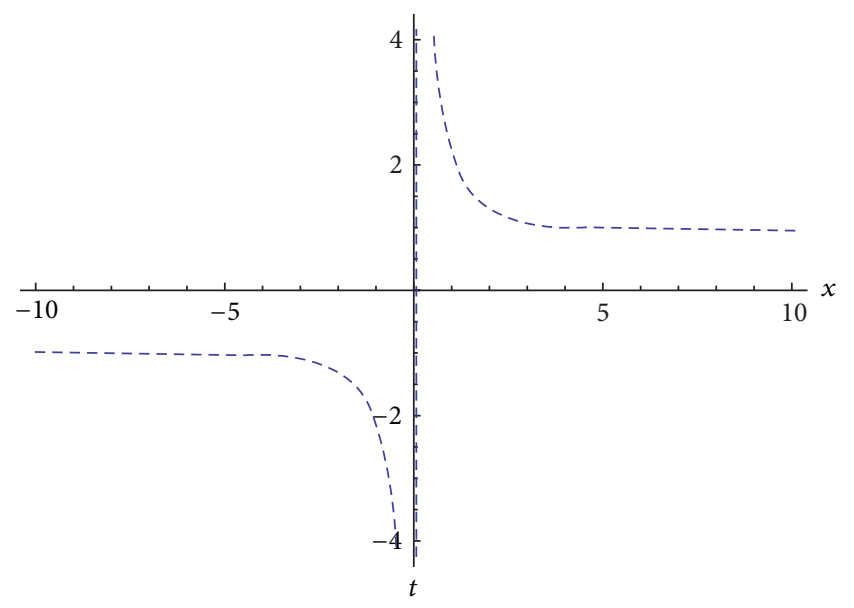

(b)

FIgURE 2: The solution (39) is shown at $k=w=1$, and the second graph represents the exact analytical solution of (39) for $t=0.5$.

where $\lambda=1$, and in order to obtain Burger-like solution of equation, we get

$$
u(x, t)=u(\xi), \quad \xi=k(x-w t) .
$$

Substituting (27) into (26), we get

$$
-w u^{\prime}+u^{\prime}+u u^{\prime}+k u^{\prime \prime}=0
$$

Balancing $\left(u u^{\prime}\right)$ with $u^{\prime \prime}$ gives $m=1$. Therefore, we may choose the following ansatz:

$$
u=a_{0}+a_{1} F \text {. }
$$

Substituting (29) into (28) along with (5) and using Mathematica yield the following set of algebraic polynomials with respect to unknowns $a_{0}, a_{1}, a_{2}$, and $a_{3}$

$$
\begin{gathered}
u^{\prime}=a_{1} A+a_{1} B F+a_{1} C F^{2}+2 a_{2} A F \\
+2 a_{2} B F^{2}+2 a_{2} C F^{3}=0, \\
u u^{\prime}=\left(a_{0}+a_{1} F\right)\left(a_{1} A+a_{1} B F+a_{1} C F^{2}\right), \\
u^{\prime \prime \prime}=a_{1} B A+a_{1} B^{2} F+a_{1} C A F \\
\quad+3 a_{1} B C F^{2}+2 a_{1} C^{2} F^{3}, \\
F^{0}: w^{2} a_{1} A-a_{1} A+2 a_{0} a_{1} A-k^{2} w^{2} a_{1} B^{2} A \\
-2 k^{2} w^{2} a_{1} A^{2} C-6 k^{2} w^{2} a_{2} A^{2} B=0,
\end{gathered}
$$




$$
\begin{aligned}
F^{1}: & w^{2} a_{1} B+2 w^{2} a_{2} A-a_{1} B-2 a_{2} B+2 a_{0} a_{1} B \\
& +4 a_{0} a_{2} A+2 a_{1}^{2} A-k^{2} w^{2} a_{1} B^{3} \\
& -8 k^{2} w^{2} a_{1} A B C-14 k^{2} w^{2} a_{2} A B^{2} \\
& -16 k^{2} w^{2} a_{2} A^{2} C=0, \\
F^{2}: & w^{2} a_{1} C+2 w^{2} a_{2} B-a_{1} C-2 a_{2} B+2 a_{0} a_{1} C \\
& +4 a_{0} a_{2} B+2 a_{1}^{2} B+6 a_{1} a_{2} A-7 k^{2} w^{2} a_{1} B^{2} C \\
& -8 k^{2} w^{2} a_{1} A C^{2}-52 k^{2} w^{2} a_{2} B^{3}=0, \\
F^{3}: & 2 w^{2} a_{2} C-2 a_{2} C+4 a_{0} a_{2} C+2 a_{1}^{2} C+4 a_{1} a_{2} B \\
& +2 a_{1} a_{2} B+4 a_{2}^{2} A-12 k^{2} w^{2} a_{1} B C^{2} \\
& -40 k^{2} w^{2} a_{2} A C^{2}-38 k^{2} w^{2} B^{2} C=0 .
\end{aligned}
$$

From the solutions of the system, we can find

$$
a_{0}=w-1, \quad a_{1}=-2 k c,
$$

and we obtain the following multiple solution and triangular periodic solutions of (26).

(i) When we choose $A=C=1, B=0$ in (31), then

$$
a_{1}=-2 k, \quad a_{0}=w-1 .
$$

Therefore, the solution can be found as

$$
u(x, t)=(w-1)-2 k \tan [k(x-w t)] .
$$

(ii) In the case if we take $A=C=-1, B=0$ in (31), then we have

$$
\begin{gathered}
a_{1}=2 k, \quad a_{0}=w-1, \\
u(x, t)=(w-1)+2 k \cot [k(x-w t)] .
\end{gathered}
$$

(iii) Again, when we choose $A=1, B=0$, and $C=-1$,

$$
\begin{gathered}
a_{0}=w-1, \quad a_{1}=-2 k, \\
u(x, t)=(w-1)+2 k \tanh [k(x-w t)], \\
u(x, t)=(w-1)+2 k \operatorname{coth}[k(x-w t)] .
\end{gathered}
$$

(iv) When we choose $A=(1 / 2), B=0$, and $C=-(1 / 2)$,

$$
\begin{gathered}
a_{0}=w-1, \quad a_{1}=-k, \\
u(x, t)=(w-1)-k[\sec [k(x-w t)]+\tan [k(x-w t)]], \\
u(x, t)=(w-1)-k[\operatorname{cosec}[k(x-w t)]+\cot [k(x-w t)]] .
\end{gathered}
$$

(v) When we choose $A=C=-(1 / 2), B=0$, then we can find the coefficients of (31) as follows:

$$
\begin{gathered}
a_{0}=k, \quad a_{0}=w-1, \\
u(x, t)=(w-1)+k[\sec [k(x-w t)]-\tan [k(x-w t)]], \\
u(x, t)=(w-1)+k[\operatorname{cosec}[k(x-w t)]-\cot [k(x-w t)]] .
\end{gathered}
$$

(vi) When we choose $A=(1 / 2), B=0$, and $C=-(1 / 2)$, then we can find the coefficients of (31) as follows:

$$
\begin{aligned}
& u(x, t)=(w-1)+k[\operatorname{coth}[k(x-w t)] \pm \operatorname{cosech}[k(x-w t)]], \\
& u(x, t)=(w-1)+k[\tanh [k(x-w t)] \pm i \operatorname{sech}[k(x-w t)]] .
\end{aligned}
$$

Figure 2 gives to us 2D and 3D graphics for (39).

\section{Conclusion}

We have presented a generalized tanh function method and used it to solve the Boussinesq equation and the Burgers-like equation. In fact this method is readily applicable to a large variety of nonlinear PDEs.

Firstly, all the nonlinear PDEs which can be solved by other tanh function method can be solved easily by this method. Secondly we have used only the special solutions of (4). If we use only the special solutions of (4), we can obtain more solutions. We are also aware of the fact that not all fundamental equations can be treated with the method.

We also obtain some new and more general solutions at the same time. Furthermore, this method is also computerizable, which allows us to perform complicated and tedious algebraic calculation on a computer.

\section{References}

[1] B. R. Duffy and E. J. Parkes, "Travelling solitary wave solutions to a seventh-order generalized KdV equation," Physics Letters A, vol. 214, no. 5-6, pp. 271-272, 1996.

[2] E. J. Parkes and B. R. Duffy, "Travelling solitary wave solutions to a compound KdV-Burgers equation," Physics Letters A, vol. 229, no. 4, pp. 217-220, 1997.

[3] Z. B. Li, "Exact solitary wave solutions of nonlinear evolution equations," in Mathematics Mechanization and Application, X. S. Gao and D. M. Wang, Eds., Academic Press, 2000.

[4] Z. B. Li and M. L. Wang, "Travelling wave solutions to the twodimensional KdV-Burgers equation," Journal of Physics A, vol. 26, no. 21, pp. 6027-6031, 1993.

[5] E. J. Parkers, "Exact solutions to the two-dimensional Korteweg-de Vries-Burgers equation," Journal of Physics A, vol. 27, pp. L497-L501, 1994.

[6] W. Hereman, P. P. Banerjee, A. Korpel, G. Assanto, A. Van Immerzeele, and A. Meerpoel, "Exact solitary wave solutions of nonlinear evolution and wave equations using a direct algebraic method," Journal of Physics A, vol. 19, no. 5, pp. 607-628, 1986.

[7] A. H. Khater, M. A. Helal, and O. H. El-Kalaawy, "Bäcklund transformations: exact solutions for the KdV and the CalogeroDegasperis-Fokas mKdV equations," Mathematical Methods in the Applied Sciences, vol. 21, no. 8, pp. 719-731, 1998.

[8] S. A. Elwakil, S. K. El-labany, M. A. Zahran, and R. Sabry, "Modified extended tanh-function method for solving nonlinear partial differential equations," Physics Letters A, vol. 299, no. 2-3, pp. 179-188, 2002.

[9] Y. Lei, Z. Fajiang, and W. Yinghai, “The homogeneous balance method, Lax pair, Hirota transformation and a general fifthorder KdV equation," Chaos, Solitons and Fractals, vol. 13, no. 2, pp. 337-340, 2002. 
[10] J. F. Zhang and Y. G. Shen, "Quantum cosmology with a complex $\varphi 4$ field at finite temperature," International Journal of Theoretical Physics, vol. 38, no. 7, pp. 1969-1979, 1999.

[11] M. Wang, "Exact solutions for a compound KdV-Burgers equation," Physics Letters A, vol. 213, no. 5-6, pp. 279-287, 1996.

[12] M. L. Wang, Y. B. Zhou, and Z. B. Li, "Application of a homogeneous balance method to exact solutions of nonlinear equations in mathematical physics," Physics Letters A, vol. 216, no. $1-5$, pp. 67-75, 1996.

[13] H. Bulut and E. Turhan, "New multiple solution to the shallow water wave equation," E-Journal of New World Sciences Academy, vol. 1, no. 4, pp. 103-106, 2006.

[14] E. J. Parkers and B. R. Duffy, "An automated tanh-function method for finding solitary wave solutions to non-linear evolution equations," Computer Physics Communications, vol. 98, no. 3, pp. 288-300, 1996.

[15] L. Yang, J. B. Liu, and K. Q. Yang, "Exact solutions of nonlinear PDE, nonlinear transformations and reduction of nonlinear PDE to a quadrature," Physics Letters A, vol. 278, no. 5, pp. 267270, 2001.

[16] F. Xie, Z. Yan, and H. Zhang, "Explicit and exact traveling wave solutions of Whitham-Broer-Kaup shallow water equations," Physics Letters A, vol. 285, no. 1-2, pp. 76-80, 2001.

[17] E. G. Fan, "Extended tanh-function method and its applications to nonlinear equations," Physics Letters A, vol. 277, no. 4-5, pp. 212-218, 2000.

[18] H. Chen and H. Zhang, "New multiple soliton solutions to the general Burgers-Fisher equation and the Kuramoto-Sivashinsky equation," Chaos, Solitons and Fractals, vol. 19, no. 1, pp. 71-76, 2004. 


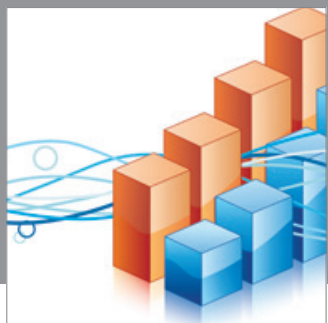

Advances in

Operations Research

mansans

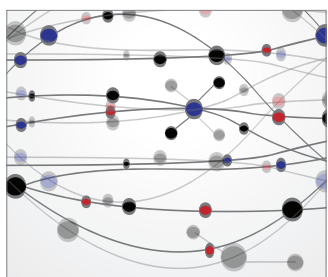

The Scientific World Journal
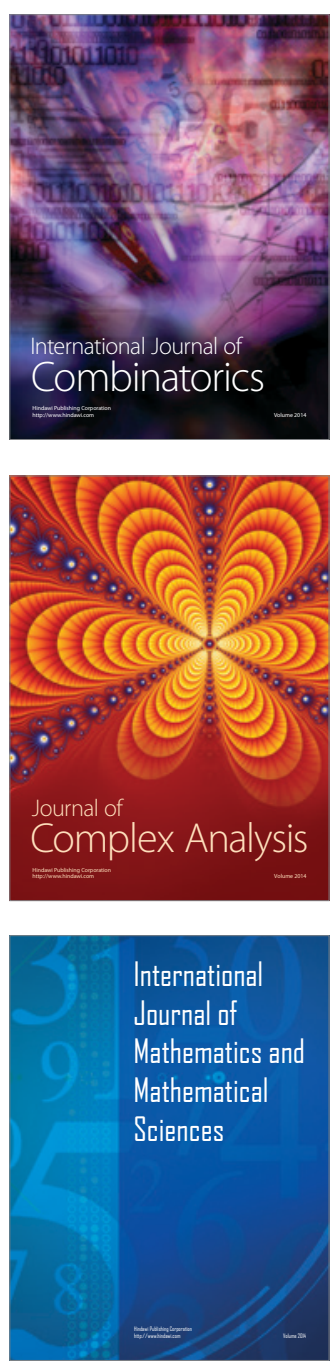
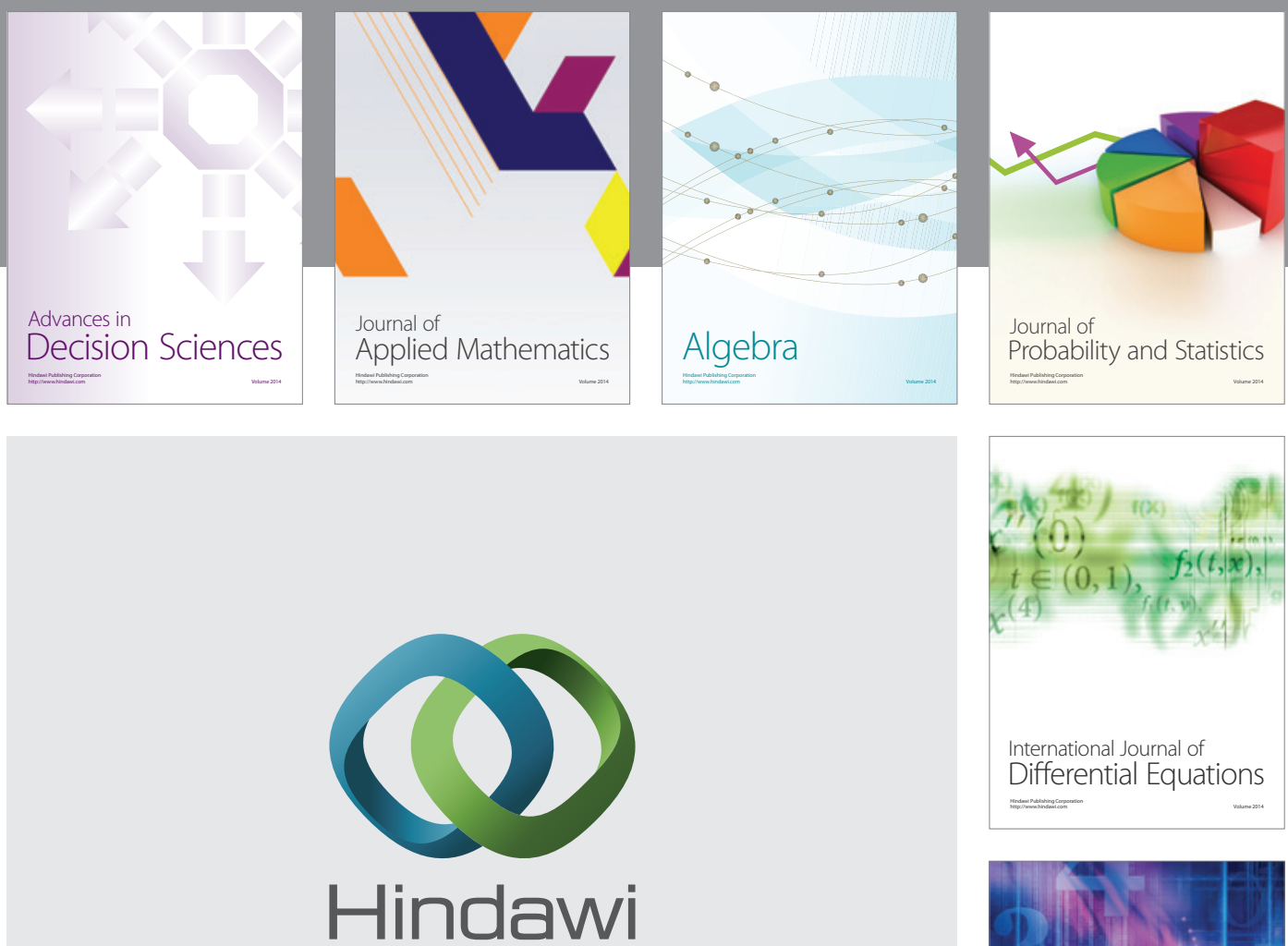

Submit your manuscripts at http://www.hindawi.com
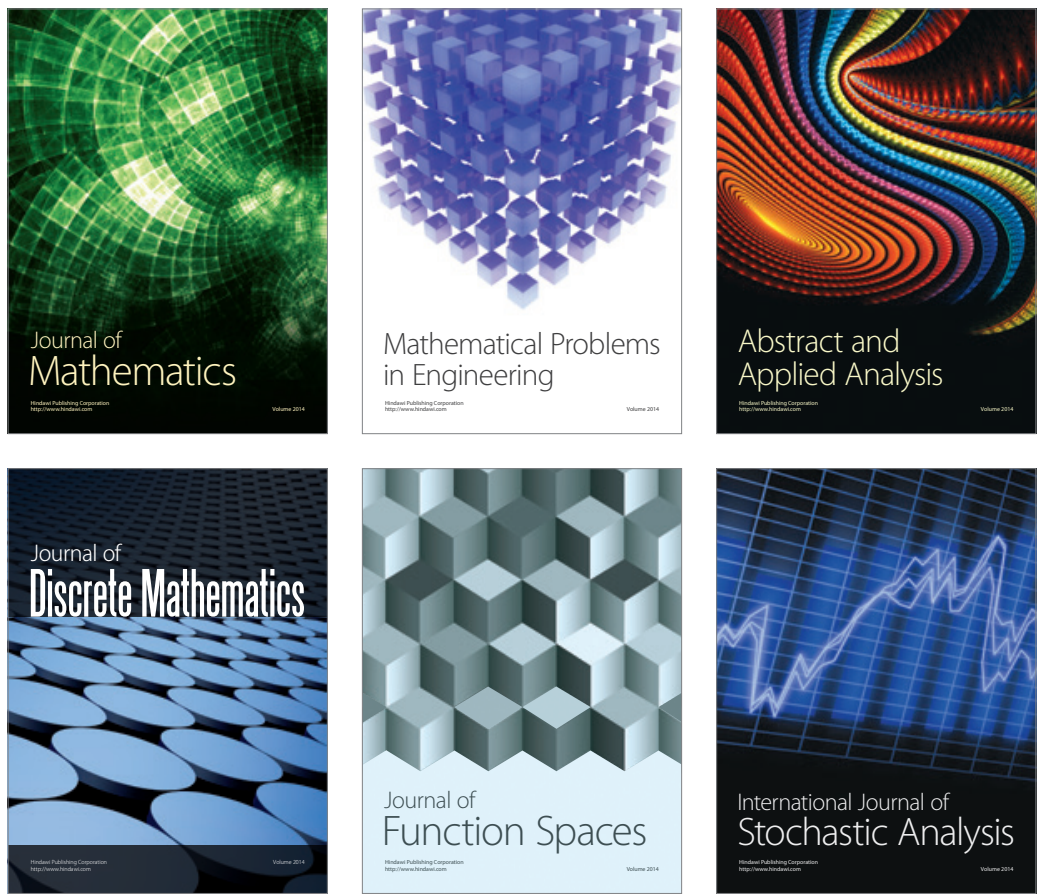

Journal of

Function Spaces

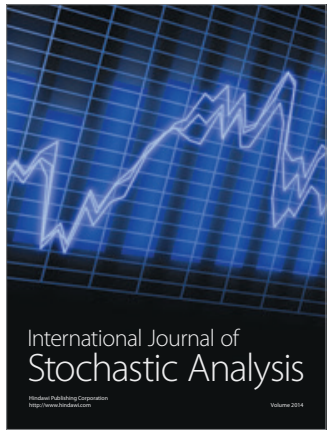

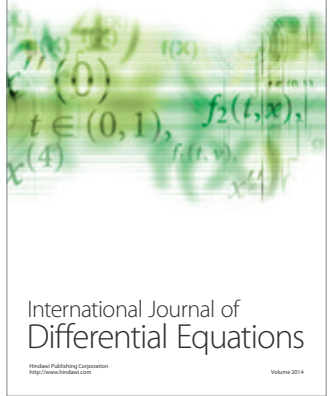
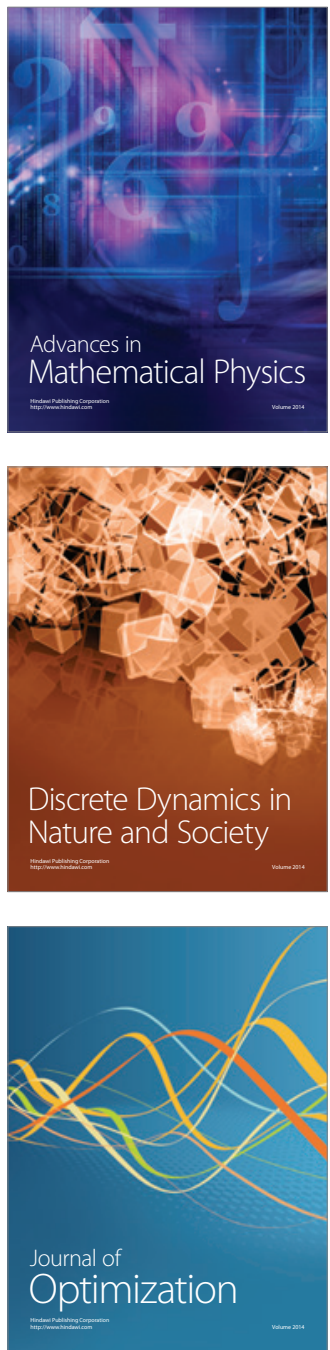